\title{
Texture analysis of early cerebral tissue damage in magnetic resonance imaging of patients with lung cancer
}

\author{
JIYING XU $^{1^{*}}$, XIAOXIAO CUI ${ }^{2 *}$, BOMIN WANG $^{2}$, GUANGYU WANG $^{1}$, MENG HAN $^{1}$, \\ RANRAN LI ${ }^{1}$, YANA QI ${ }^{1}$, JIANJUN XIU ${ }^{1}$, QIANLONG YANG ${ }^{1}$, ZHI LIU ${ }^{2}$ and MINGYONG HAN ${ }^{1}$ \\ ${ }^{1}$ Cancer Therapy and Research Center, Shandong Provincial Hospital Affiliated to Shandong University, Jinan, \\ Shandong 250021; ${ }^{2}$ School of Information Science and Engineering, Shandong University, Jinan, Shandong 250100, P.R. China
}

Received January 26, 2019; Accepted October 23, 2019

DOI: $10.3892 / \mathrm{ol} .2020 .11426$

\begin{abstract}
Primary tumors can secrete many cytokines, inducing tissue damage or microstructural changes in distant organs. The purpose of this study was to investigate changes in texture features in the cerebral tissue of patients with lung cancer without brain metastasis. In this study, 50 patients with lung cancers underwent 3.0-T magnetic resonance imaging (MRI) within 2 weeks of being diagnosed with lung cancer. Texture analysis (TA) was carried out in 8 gray matter areas, including bilateral frontal cortices, parietal cortices, occipital cortices and temporal cortices, as well as 2 areas of bilateral frontoparietal white matter. The same procedure was performed for 57 healthy controls. A total of 32 texture parameters were separately compared between the patients and controls in the different cerebral tissue sites. Texture features among patients based on histological type and clinical stage were also compared. Of the 32 texture parameters, 27 showed significant differences between patients with lung cancer and healthy controls. There were significant differences in cerebral tissue, both gray matter and white matter between patients and controls, especially in several wavelet-based parameters. However, there were no significant differences between tissue at homologous sites in bilateral hemispheres, either in patients or controls. TA detected overt changes in the texture features of cerebral tissue in patients with lung cancer without brain
\end{abstract}

Correspondence to: Professor Mingyong Han, Cancer Therapy and Research Center, Shandong Provincial Hospital Affiliated to Shandong University, 324 Jingwuweiqi Road, Jinan, Shandong 250021, P.R. China

E-mail: hanmingyong@sina.com

Professor Zhi Liu, School of Information Science and Engineering, Shandong University, 27 Shanda South Road, Jinan, Shandong 250100, P.R. China

E-mail: liuzhi@sdu.edu.cn

*Contributed equally

Key words: lung cancer, magnetic resonance imaging, texture analysis, cerebral tissue metastasis compared with those of healthy controls. TA may be considered as a novel and adjunctive approach to conventional brain MRI to reveal cerebral tissue changes invisible on MRI alone in patients with lung cancer.

\section{Introduction}

According to the 2018 Global Cancer Statistics, lung cancer is the leading cause of cancer-associated mortality worldwide (1) and was so in China in 2014 (2). However, the cure for lung cancer remains elusive due to the aggressive and disseminative nature of tumors which restricts the efficacy of cancer treatments (3). The brain is a preferential site of metastasis in lung cancer (4). Among patients with lung cancer, $30-50 \%$ develop brain metastasis (5-7), which substantially affects their quality of life. However, in the present study, even in the absence of brain metastasis, some individuals continued to exhibit paraneoplastic neurological syndromes, such as behavioral, memory and movement disorders as well as hypomnesis, dizziness and headaches (8). A number of previous studies have hypothesized that the pathophysiology underlying paraneoplastic syndromes is associated with the secretion of cytokines by the primary tumor (9-12). This may induce injury to distant organs and cause microstructural changes like those in the brain, which cause paraneoplastic syndromes or alter the homing and proliferation of metastatic cancer cells (9-13). However, the underlying mechanisms remain unclear.

Due to the potential ability to assess the characteristics of brain tissue noninvasively, magnetic resonance imaging (MRI) is currently the main method used for diagnosis, treatment guidance and monitoring of brain metastasis $(14,15)$. However, identifying early changes in the cerebral tissue of patients with lung cancer without brain metastasis is difficult (16). Fortunately, MRI images of cerebral tissues contain a wealth of information invisible to the human eye that can accessed by texture analysis (TA) techniques (17). Texture is a visual stimulus generated by repetitive image patterns, which can be depicted as regular or irregular, smooth or rough and fine or coarse. Although they can exhibit very complex patterns, some textures can be extracted relatively easily even by visual assessment due to the regular visual appearance of the patterns. However, most textures display random patterns because textural primitives are random, making them difficult 
to recognize and interpret. In medical images, these types of random textures are more common than regular patterns. As texture is an image feature defined by both pixel locations and brightness value, TA enables mathematical calculation of the patterns. Therefore, texture features provide the potential to distinguish and characterize properties of cerebral tissue (17).

The aim of the present study was to use texture analysis (TA) methods to describe early cerebral tissue damage caused by lung cancer without brain metastasis.

\section{Materials and methods}

Subjects. The present study was approved by the Ethics Committee of Shandong Provincial Hospital Affiliated to Shandong University (Jinan, China). Written informed consent was obtained from all patients prior to the study start. Patients with lung cancer were recruited from the Shandong Provincial Hospital between December 2017 and October 2018. The TA study included 50 consecutive patients (36 males, 14 females; mean age, 60 years; age range 47-75 years) who were all diagnosed by pathology and met the diagnostic criteria for primary lung cancer according to the World Health Organization (18). In addition to a detailed review of pathological specimens by pathologists upon admission to the hospital, all patients with lung cancer underwent a careful physical examination performed by experienced oncologists and neurologists from Shandong Provincial Hospital Affiliated to Shandong University, in order to determine the clinical stage, Karnofsky Performance Scale (KPS) score (19) and numeric rating scale score (NRS) (20). The demographic information and clinical characteristics of the patients are presented in Table I. The inclusion criteria were as follows: i) Age, 18-75 years; ii) histological type of small cell carcinoma, squamous cell carcinoma, adenocarcinoma and others; iii) KPS score $>60$ and iv) no neurological abnormalities. The exclusion criteria were as follows: i) Age, $<18$ years or $>75$ years; ii) systemic illness such as systemic lupus erythematosus, serious vascular disease, head trauma, epilepsy (healthy controls) or claustrophobia; iii) long-term use of psychoactive medication and iv) refusal to undergo MRI. The control group (CG) comprised of 57 ageand sex-matched healthy volunteers. The individuals in this group reported no types of malignant tumor or any systemic, neurological or psychiatric illnesses known to affect cerebral structure or function such as a long history of psychoactive medication use, head trauma, serious vascular disease, current depression, epilepsy or alcoholism.

MRI examinations. Data were acquired within 2 weeks of diagnosis of lung cancer primarily due to some patients being diagnosed in other hospitals and then transferred to Shandong Provincial Hospital for further antitumor treatment. Following diagnosis, all patients underwent conventional MRI using a 3.0-T scanner (Philips Medical Systems B.V.) equipped with an 8-channel head and neck coil. The MRI machine was included in a detailed quality control plan requiring daily, monthly and quarterly inspections. The radio frequency amplifier properties and main magnetic field homogeneity of the MRI machine were measured and controlled quarterly. The following conventional MRI sequences were employed: Sagittal, coronal and axial T2-weighted imaging (T2WI), T1-weighted imaging
Table I. Clinical characteristics of the studied groups.

\begin{tabular}{lcc}
\hline Characteristics & $\begin{array}{c}\text { Patient, } \\
\mathrm{n}=50\end{array}$ & $\begin{array}{c}\text { Control, } \\
\mathrm{n}=57\end{array}$ \\
\hline $\begin{array}{l}\text { Age in years, mean } \pm \text { standard } \\
\text { deviation }\end{array}$ & $60.0 \pm 7.2$ & $59.1 \pm 6.5$ \\
Age range, years & $47-75$ & $48-75$ \\
Gender, Female vs. Male & $36 / 14$ & $37 / 20$ \\
Histological type, $\mathrm{n}$ & & \\
$\quad$ Adenocarcinoma & 29 & - \\
Squamous & 17 & - \\
$\quad$ Small cell & 4 & - \\
Clinical stage & & - \\
$\quad$ Early & 18 & - \\
Advanced & 32 & - \\
Karnofsky performance scale & & - \\
$\geq 70$ & 49 & - \\
$<70$ & 1 & - \\
Numeric rating scales & & - \\
$\leq 3$ & 37 & \\
$>3$ & 13 & \\
\hline
\end{tabular}

(T1WI), fluid-attenuated inversion recovery imaging, diffusion-weighted imaging, apparent diffusion coefficient and enhanced T1WI. The sequences employed in the MRI protocol are presented in Table II.

MR image segmentation. Image selection was carried out using a DICOM viewer (version 3.0; Philips Medical Systems B.V.). For each MR image, regions of interest (ROIs) were drawn symmetrically by hand on bilateral sides of the hemispheres at each level of interest $(21,22)$. In the present study, 16x16-pixel ROIs were manually drawn on the frontoparietal white matter in the centrum semiovale (Fig. 1A), frontal cortices and parietal cortices at the level of the body of the lateral ventricles (Fig. 1B), occipital cortices at the level of the basal ganglia (Fig. 1C) and temporal cortices at the level of the midbrain (Fig. 1D). The ROIs were carefully placed by two experienced neuroradiologists from Shandong Provincial Hospital Affiliated to Shandong University, to avoid overlap with any areas of microhemorrhage, hyperintensity or macroscopic hemosiderin depositions which may occur in some patients. Fig. 2 presents an array of texture blocks extracted from the cerebral tissues of a patient with lung cancer, without metastasis and a healthy volunteer.

TA analysis technique. The axial T2WI sequence was selected from the whole MRI study for TA as T2-weighted MR imaging has been demonstrated to be sensitive to tissue abnormalities in the human brain (23). Only single slices were evaluated to obtain a maximum in-plane resolution by avoiding the lower resolution or interpolation, typically caused by 3 -dimensional TA (24). Further analysis was performed using 4 MRI image slices selected from the T2WI sequence at 4 interest levels. The texture features in 10 ROIs were assessed based on the histogram, gray-level co-occurrence matrix (GLCM), 
Table II. MRI sequences included in the magnetic resonance protocol of the present study.

\begin{tabular}{lccccccc}
\hline Sequence & TR & TE & TI & Slice/gap & Matrix & FOV & Flip angle \\
\hline Axial T2WI & 4,000 & 100 & 0 & $6.0 / 1.0$ & $384 \times 384$ & 230 & 90 \\
Axial TIWI-IR & 3,000 & 43 & 1,150 & $6.0 / 1.0$ & $264 \times 264$ & 230 & - \\
Axial FLAIR & 9,000 & 148 & 2,500 & $6.0 / 1.0$ & $284 \times 284$ & 230 & - \\
DWI & 2,257 & 71 & 0 & $6.0 / 1.0$ & $152 \times 152$ & 230 & 90 \\
Axial T1W-STIR & 3,000 & 43 & 1,150 & $6.0 / 1.0$ & $264 \times 264$ & 230 & -
\end{tabular}

TR, repetition time; TE, echo time; TI, inversion time; FOV, field of view; T1WI, T1-weighted imaging; T2WI, T2-weighted imaging; DWI, diffusion-weighted imaging; FLAIR, fluid-attenuated inversion recovery; IR, inverse recovery; STIR, short time inversion recovery.
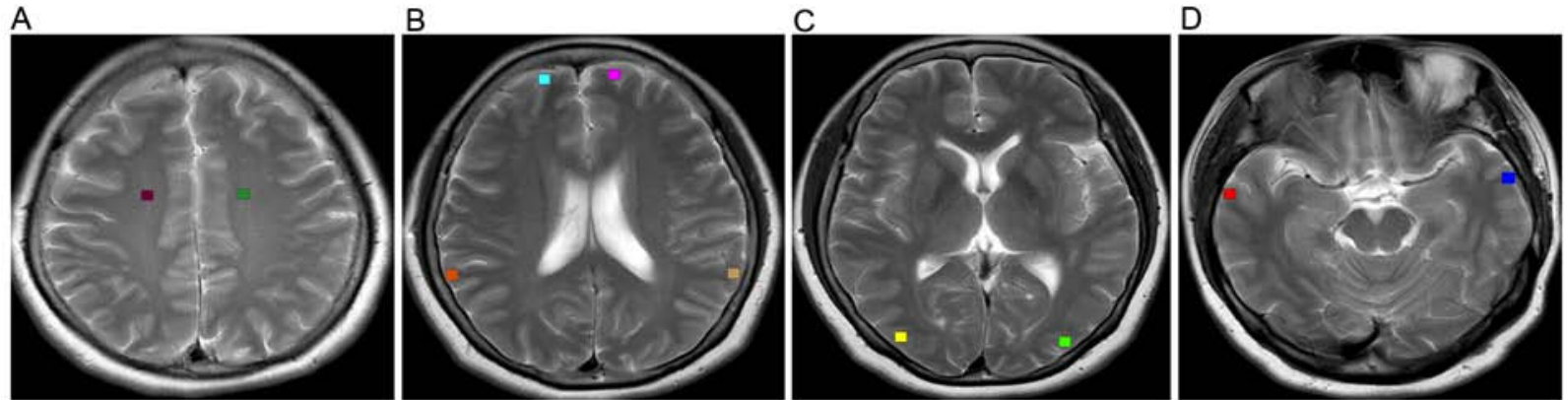

Figure 1. ROIs delineated at selected levels. (A) ROIs placed in frontoparietal white matter in the centrum semiovale. (B) ROIs placed in frontal and parietal cortices at the level of the body of the lateral ventricles. (C) ROIs placed in occipital cortices at the level of the basal ganglia. (D) ROIs placed in the temporal cortices at the level of midbrain. ROIs, regions of interest.
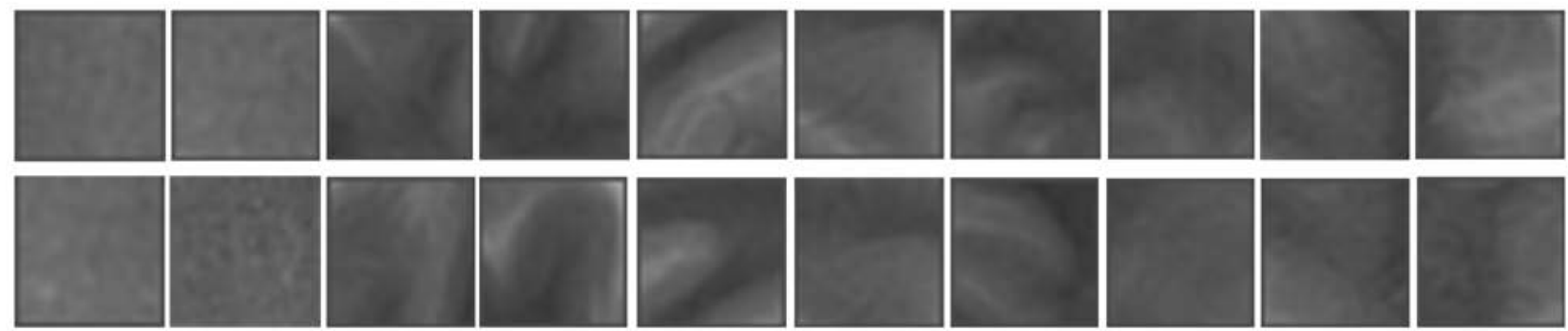

Figure 2. Examples of textures. Top row, the brain of a patient with lung cancer, without metastasis; bottom row, a healthy brain. Each block contains 16x16 pixels.

gray-level run length matrix (GLRLM) and wavelet transform, as summarized in Table III $(17,25,26)$. The wavelet transform of the three dimensional image was the decomposition of the image along the $\mathrm{x}, \mathrm{y}$ and $\mathrm{z}$ directions using low-pass filter (L) and high-pass filter $(\mathrm{H})$, and each layer of wavelet decomposition decomposed the image data into eight different frequency bands (LLH, HLL, HLH, HHL, LHL, LHH, HHH and LLL), which included seven high frequency bands (LLH-HHH) and one low frequency band (LLL).

The first order statistics image properties derived from the gray-scale frequency histogram depend solely on individual pixel values. Second order statistics are properties of pixel pairs and describe the relationship between pixels and their grey levels. However, the first and second order statistics of images in texture analysis only contained the amplitude information of the image, ignoring the phase information, thus the wavelet transform of the image was also analyzed. TA was performed using the Python language (version 3.6) on the JetBrains PyCharm platform (version 2018.1.3). Feature calculations were performed within the Pyradiomics package (version 2.2.0), which is an open-source Python package designed for the extraction of radiomic features from medical images (27). As the Fisher criterion can produce an array of features with highly discriminative power, a feature selection method was applied using the Fisher coefficient provided by the Python package, in order to identify the six texture features with the highest discriminatory potential for classification (17).

Statistical analysis. In total, 32 texture parameters were tested to determine which and how many features in each ROI demonstrated significant differences between patients with lung cancer, without brain metastasis and healthy controls. Statistical analyses were performed for each texture feature. Differences in texture features between the two groups were analyzed via the Wilcoxon rank sum test or Mann-Whitney U test, using SPSS software (version 22; IBM Corp.). The Fisher 
Table III. List of texture features.

\begin{tabular}{llll}
\hline Histogram & Gray-level co-occurrence matrix & Gray-level run length matrix & Wavelet \\
\hline Mean & Joint energy & Long run emphasis & HH_kurtosis \\
Skewness & Inverse difference & Run length non-uniformity & HLH_skewness \\
Deviation & Correlation & Low gray-level run emphasis & LLL_10 percentile \\
Variance & Difference average & Short run low gray-level emphasis & LLL_median \\
Kurtosis & Difference entropy & Long run low gray-level emphasis & LLL_minimum \\
& Inverse difference normalized & Short run high gray-level emphasis & LLL_variance \\
& Joint entropy & Long run high gray-level emphasis & \\
& Sum average & Short run emphasis & \\
& Sum entropy & Run gray-level non-uniformity &
\end{tabular}

LLL, low frequency-low frequency-low frequency output; HHH, high frequency-high frequency-high frequency output; HHL, high frequencyhigh frequency-low frequency output; HLH, high frequency-low frequency-high frequency output.

coefficient was used to select the six most discriminative parameters. $\mathrm{P}<0.05$ was considered to indicate a statistically significant difference.

\section{Results}

Analysis workflow. Given brain MRI data sets of patients with lung cancer without brain metastasis and healthy controls, four interest levels were selected, and then texture blocks of $16 \times 16$ pixels were manually placed in a symmetrical manner on bilateral sides of the hemispheres at each level of interest. Finally, texture features were calculated based on the image histogram, the co-occurrence matrix, the run-length matrix and wavelets. The system diagram presented in Fig. 3 represents the process followed for MRI analysis.

Brain MRI of patients with lung cancer and healthy controls. In this study, conventional MRI enabled detection of lesions related to brain metastasis or primary brain tumors only when gross structural abnormalities could be visually detected. Based on the conventional MR images, no significantly different signals were found in any sequence in patients with lung cancer without metastasis compared with healthy volunteers (Table II). Fig. 4 shows conventional MR images of a 45-year-old female patient who presented with lung cancer without brain metastasis (top row) and a 54-year-old male healthy control (bottom row).

Comparison of texture parameters in bilateral hemispheres between patients with lung cancer without brain metastasis and healthy controls. For the comparison of bilateral hemispheres of 10 ROIs, 32 features were tested for each texture block to determine which and how many of the parameters differed statistically between patients with primary lung cancer without brain metastasis and healthy controls. The number of texture parameters $(n=32)$ which were significantly different were analyzed using the Wilcoxon test (Table IV). The feature vector included mean and variance features from histogram statistics, energy and correlation features from a co-occurrence matrix, short-run and long-run emphasis features from a run-length matrix and phase information features from wavelet transform. The 32 texture parameters included; Five histogram-based parameters, nine GLCM-based parameters, 11 GLRLM-based parameters and seven wavelet-based parameters. Among the five histogram-based parameters, which depended solely on individual pixel gray-level values, the following four parameters; mean, skewness, deviation and variance were significantly different $(\mathrm{P}<0.05)$, and only the kurtosis parameter demonstrated no significant difference $(\mathrm{P}=0.686)$. Among the nine GLCM-based parameters, seven parameters were significantly different; however, the parameters for correlation $(\mathrm{P}=0.435)$ and normalized inverse difference $(\mathrm{P}=0.156)$ demonstrated no significant differences. All 11 GLRLM-based parameters were significantly different $(\mathrm{P}<0.05)$. Among the seven wavelet-based parameters, five parameters were significantly different $(\mathrm{P}<0.05)$, whereas wavelet-HHH_kurtosis and wavelet-HLH_skewness failed to demonstrate statistically significant differences ( $\mathrm{P}>0.05$, respectively) (Table IV). There were 27 texture parameters with a statistically significant difference including 4 histogram-based parameters, 7 GLCM-based parameters, 11 GLRLM-based parameters and 5 wavelet-based parameters. The feature parameters with statistically significant differences were in first order features, second order features and wavelets. For instance, mean indicated the average density among the texture blocks; deviation calculated the statistical variability of density among the pixels in the blocks; sum average indicated the total number of correlated pixel pairs with the same sum in the block; gray-level non-uniformity referred to the orderliness or randomness of the pixel densities among the blocks which could denote the degree of structure, and gray-level run length emphasis measured consecutive pixels of the same density along particular orientations as another representation of structure.

Identification of the six most discriminative parameters between patients with lung cancer without brain metastasis and healthy controls. When the texture parameters of 10 ROIs were compared in bilateral hemispheres between the lung cancer group and the healthy control group, 27 of the 
Table IV. Comparison of texture features between patients with lung cancer and healthy controls.

\begin{tabular}{|c|c|c|c|}
\hline Feature & Patient, mean (SD) & Control, mean (SD) & P-value \\
\hline Kurtosis & $3.649(1.88)$ & $3.707(2.57)$ & 0.686 \\
\hline Mean & $544.176(167.18)$ & $688.647(209.26)$ & $<0.001$ \\
\hline Skewness & $0.614(0.61)$ & $0.465(0.67)$ & 0.001 \\
\hline Deviation & $64.877(42.55)$ & $78.146(47.07)$ & $<0.001$ \\
\hline Variance & $6,016.221(460.30)$ & $8,319.324(434.89)$ & $<0.001$ \\
\hline Correlation & $0.806(0.14)$ & $0.800(0.15)$ & 0.435 \\
\hline Difference average & $0.873(0.43)$ & $1.063(0.51)$ & $<0.001$ \\
\hline Difference entropy & $1.584(0.41)$ & $1.749(0.45)$ & $<0.001$ \\
\hline Inverse difference & $0.674(0.09)$ & $0.631(0.09)$ & $<0.001$ \\
\hline Inverse difference normalized & $0.941(0.01)$ & $0.939(0.01)$ & 0.156 \\
\hline Joint energy & $0.073(0.06)$ & $0.057(0.06)$ & $<0.001$ \\
\hline Joint entropy & $4.772(1.16)$ & $5.193(1.22)$ & $<0.001$ \\
\hline Sum average & $11.151(5.34)$ & $14.097(8.38)$ & $<0.001$ \\
\hline Sum entropy & $3.791(0.85)$ & $4.044(0.90)$ & $<0.001$ \\
\hline Gray-level non-uniformity & $22.665(9.16)$ & $21.697(10.55)$ & $<0.001$ \\
\hline High gray-level run emphasis & $52.799(65.99)$ & $86.091(127.81)$ & $<0.001$ \\
\hline Long run emphasis & $4.592(2.71)$ & $3.605(1.98)$ & $<0.001$ \\
\hline Long run high gray-level emphasis & $135.634(99.71)$ & $195.944(295.67)$ & $<0.001$ \\
\hline Long run low gray-level emphasis & $0.640(0.94)$ & $0.403(0.59)$ & $<0.001$ \\
\hline Low gray-level run emphasis & $0.108(0.08)$ & $0.086(0.07)$ & $<0.001$ \\
\hline Run length non-uniformity & $80.196(33.18)$ & 96.709 (34.77) & $<0.001$ \\
\hline Run percentage & $0.618(0.11)$ & $0.673(0.10)$ & $<0.001$ \\
\hline Short run emphasis & $0.694(0.10)$ & $0.741(0.09)$ & $<0.001$ \\
\hline Short run high gray-level emphasis & $43.297(60.87)$ & $71.963(107.37)$ & $<0.001$ \\
\hline Short run low gray-level emphasis & $0.067(0.04)$ & $0.058(0.04)$ & $<0.001$ \\
\hline wavelet-HHH_kurtosis & $8.636(7.28)$ & $8.702(7.19)$ & 0.932 \\
\hline wavelet-HHL_maximum & $35.940(30.20)$ & $45.996(40.29)$ & $<0.001$ \\
\hline wavelet-HLH_skewness & $-0.224(1.21)$ & $-0.263(1.34)$ & 0.930 \\
\hline wavelet-LLL_10 percentile & $1,358.451(402.93)$ & $1,689.975(524.57)$ & $<0.001$ \\
\hline wavelet-LLL_median & $1,544.083(19.83)$ & $1,927.296(585.72)$ & $<0.001$ \\
\hline wavelet-LLL_minimum & $1,257.445(375.73)$ & $1,538.135(509.09)$ & $<0.001$ \\
\hline wavelet-LLL_variance & $46,637.723(84568.84)$ & $64,229.389(80765.98)$ & $<0.001$ \\
\hline
\end{tabular}

A total of 32 texture parameters were evaluated. The parameters that demonstrated statistically significant differences between patients with lung cancer and health controls are presented in bold. $\mathrm{P}<0.05$ was considered to indicate a statistically significant difference. LLL, low frequency-low frequency-low frequency output; HHH, high frequency-high frequency-high frequency output; HHL, high frequency-high frequency-low frequency output; $\mathrm{HLH}$, high frequency-low frequency-high frequency output.

32 were significantly different. Among the 27 significantly different parameters, six texture features were identified using the Fisher coefficient provided by the Python package. The Fisher criterion could produce a set of features with a high discriminatory potential for separation and classification which were also highly associated with each other. Based on the Fisher coefficient and the values obtained by the Wilcoxon test, the six most discriminative parameters for differences between patients and healthy controls were selected. These six texture parameters were the wavelet-LLL_median, mean, wavelet-LLL_ 10 percentile, wavelet-LLL_ minimum, run percentage, and run length non-uniformity. The six most discriminative texture features were extracted from the first-order statistics, second-order statistics and wavelets.
However, the six texture features were mainly based on the wavelets and GLRLM features (Fig. 5).

Comparison of the top six texture parameters between the left and right hemispheres in patients with lung cancer without brain metastasis and healthy controls. The comparison of bilateral hemispheres of patients with lung cancer and healthy controls, proved that TA had a high discriminatory potential, but its stability needed to be further confirmed. Firstly, the difference between bilateral hemispheres in the controls was compared using the six most discriminative parameters (Table V). For these six texture parameters, there were no significant differences between the bilateral hemispheres based on the Wilcoxon test. Subsequently, the six most 


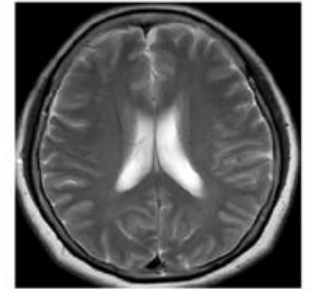

MR image
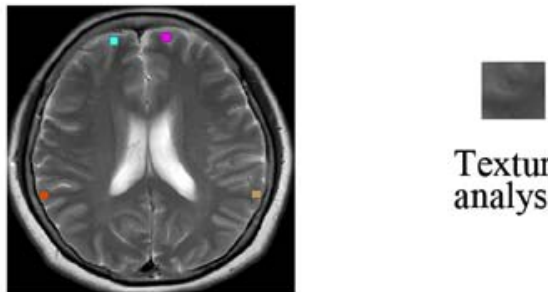

Texture analysis
Histogram

Co-occurrence matrix

Run-lengh matrix

Wavelet

Segmentation

Figure 3. Workflow for brain MR image analysis. MR, magnetic resonance.
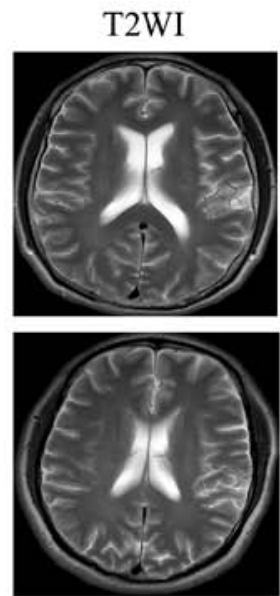

T1WI

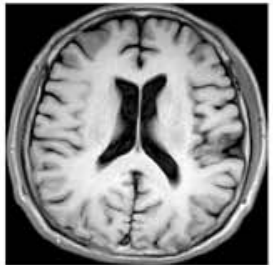

C-T1WI
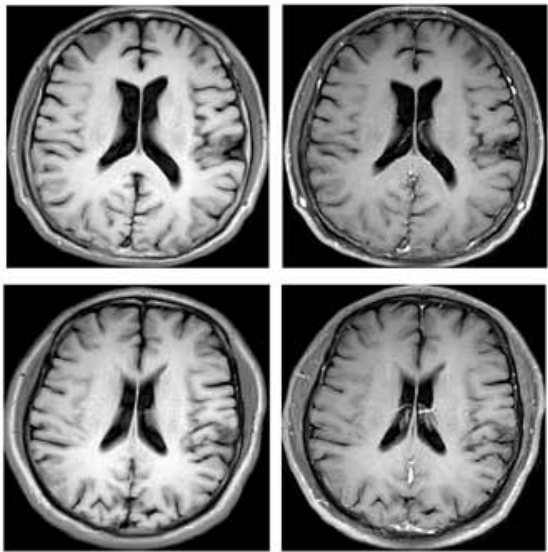
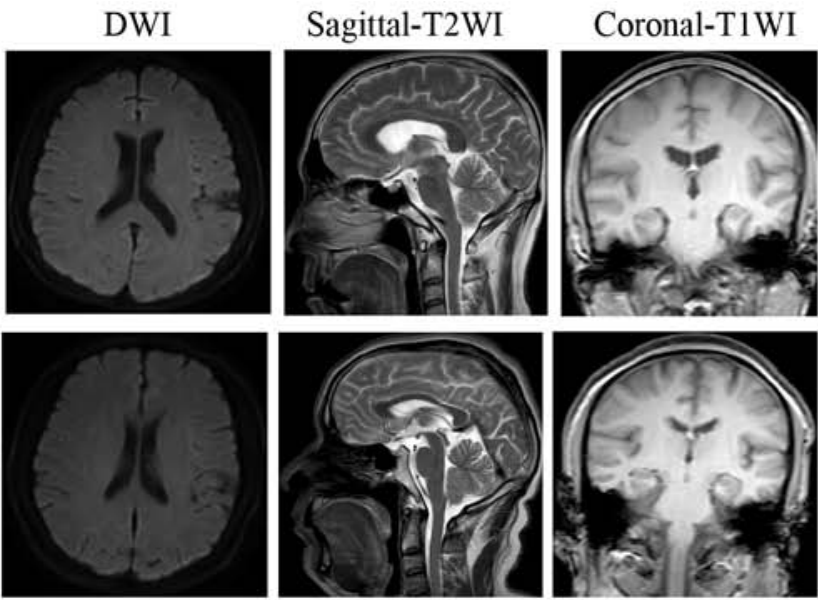

Figure 4. Different magnetic resonance sequences between patients and controls. Top row, the brain of a patient with lung cancer, without metastasis; bottom row, a healthy brain. T2WI, T2-weighted imaging; T1WI, T1-weighted imaging; C-T1WI, contrast-enhanced T1 weighted imaging; DWI, diffusion-weighted imaging.

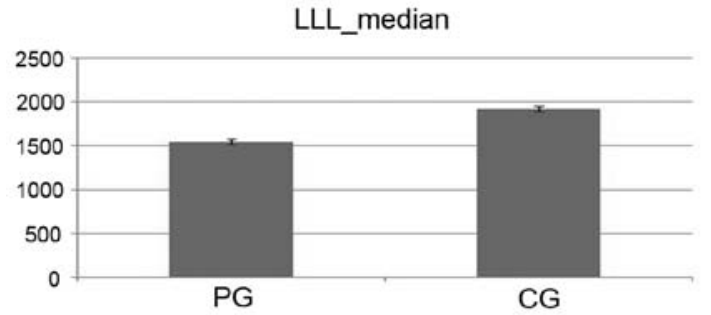

LLL_10 percentile

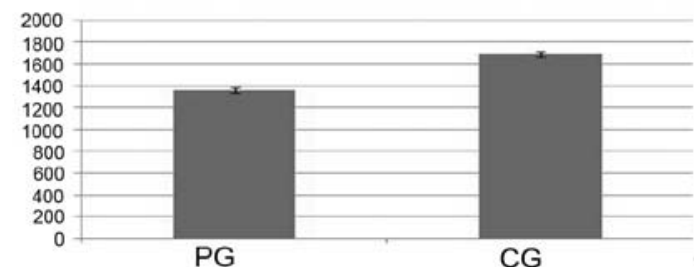

Run percentage

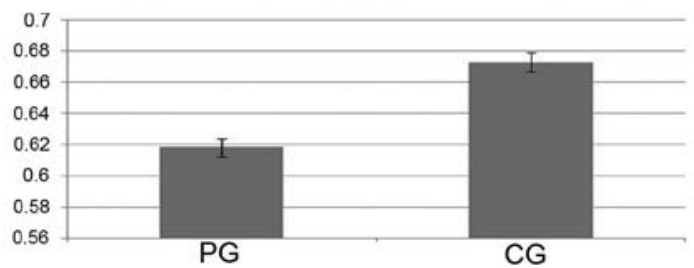

Mean

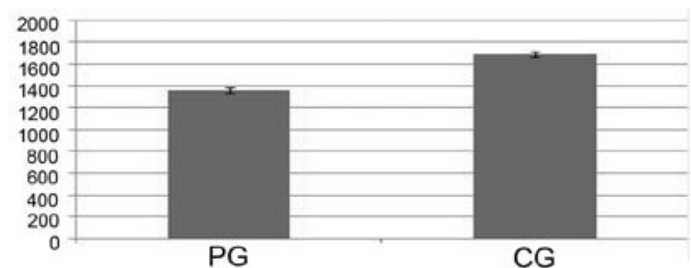

LLL_minimum

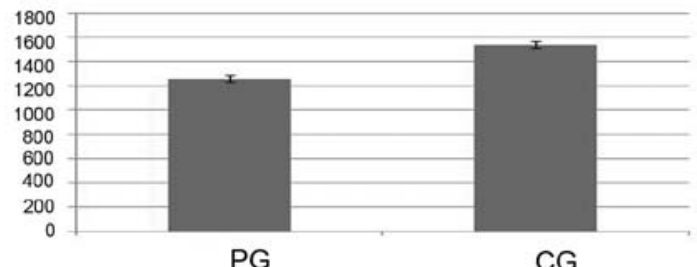

Run length non-uniformity

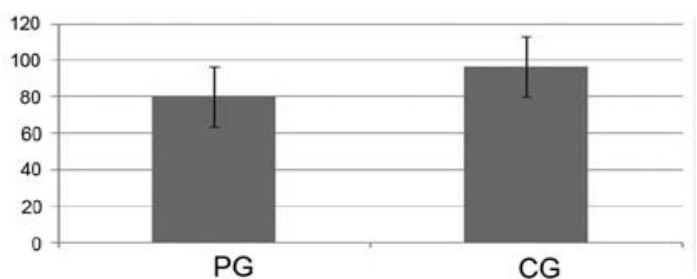

Figure 5. Comparison of different texture patterns between the PG and CG groups. The six features demonstrated significant differences between the two groups. PG, patient group; CG, control group; LLL, low frequency-low frequency-low frequency output. 
discriminative parameters were used to find out if primary lung cancer had the same impact on the bilateral hemispheres. For these six texture parameters, there were no significant differences between the bilateral hemispheres in patients with lung cancer (Table V).

Comparison of the top six texture parameters between gray and white matter in patients with lung cancer without brain metastasis and healthy controls. The top six texture parameters were tested to find out how many and which of them differed statistically between gray and white matter. The texture features of gray and white matter in patients with lung cancer and healthy controls were also analyzed using the Wilcoxon test and based on the top six texture parameters. An identical trend between gray and white matter was observed in the patient and control groups. Among the six parameters, four parameters were significantly different between the patient and control groups. These four parameters were wavelet-LLL_median, wavelet-LLL_ 10 percentile, wavelet-LLL_ minimum, and run length non-uniformity. The parameters of mean and run percentage showed no significant difference (Table VI).

Comparison of texture parameters between patients with lung cancer without brain metastasis and healthy controls in cerebral gray and white matter. The aforementioned six most discriminative parameters were used to determine which differed statistically between patients with lung cancer without brain metastasis and healthy controls in different cerebral regions. In white matter regions, all six texture parameters analyzed with Wilcoxon test showed significant differences between patients and controls (Table VII). The six most discriminative parameters were subsequently tested to determine whether primary lung cancer has the same effect on the gray matter regions. For these six texture parameters, all showed significant differences between patients and controls (Table VII).

Comparison of the texture features by clinical stage in patients with lung cancer without brain metastasis. To investigate the relationship between the texture parameters of brain MR images and clinical lung cancer stages, which was a revised 2017 edition of the American Joint Committee on Cancer (AJCC) Cancer Staging Manual $(28,29)$, patients with lung cancer, without brain metastasis were divided into two groups: Stage I+II and stage III+IV. The top six texture parameters were tested to find out how many and which of them differed statistically between the 2 patient groups. All of the six texture parameters were significantly different between the patient groups (Table VIII). Compared to the stage I+II group, all six parameters were significantly greater in the stage III+IV group (Table VIII).

Comparison of the top six texture features by histological type. The main histological types of lung cancer are squamous cell carcinoma, adenocarcinoma and small cell lung cancer (SCLC). The top six texture parameters were compared among these groups to establish associations between the brain MRI texture parameters and the histological types (Table IX). The top six texture parameters showed no significant differences between the squamous cell and adenocarcinoma groups but were all significantly different between the SCLC group

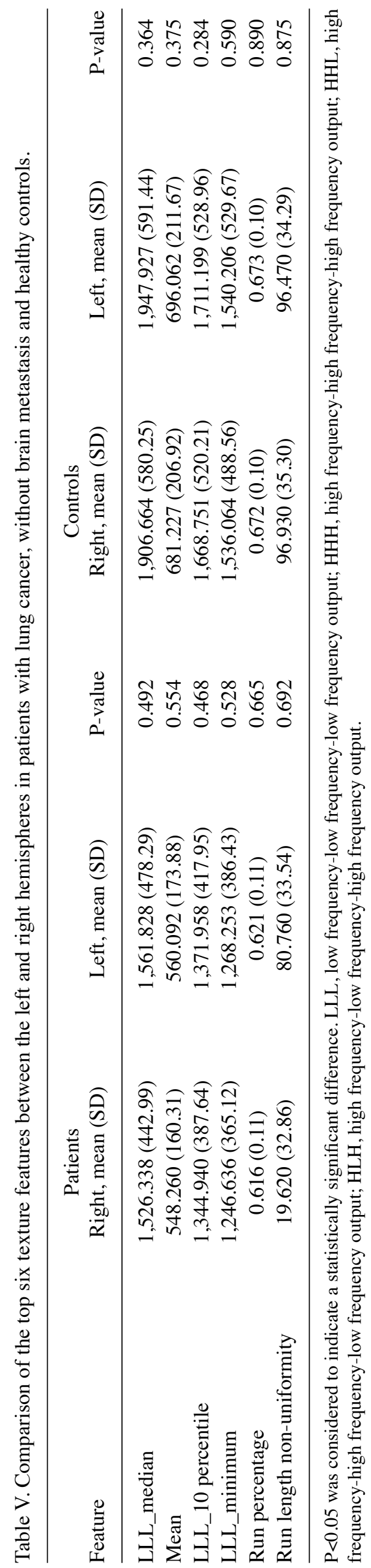



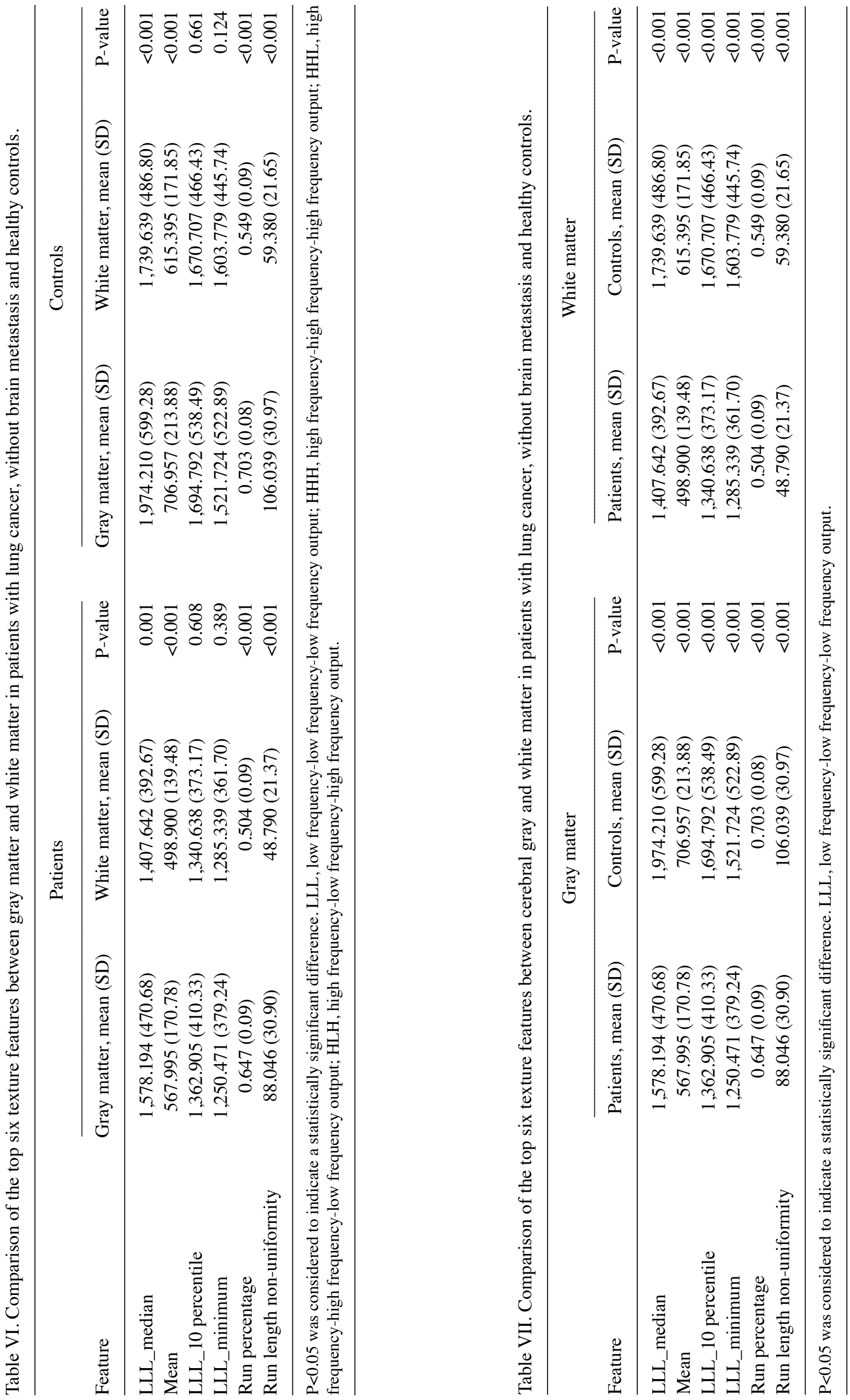
Table VIII. Comparison of the top six texture features between different clinical stages in patients with lung cancer, without brain metastasis.

\begin{tabular}{lccc}
\hline Feature & Stage I+II, mean (SD) & Stage III+IV, mean (SD) & P-value \\
\hline LLL_median & $1,348.190(196.51)$ & $1,649.503(525.46)$ & $<0.001$ \\
Mean & $482.068 .995(72.08)$ & $592.987(190.12)$ & $<0.001$ \\
LLL_10 percentile & $1,181.089(183.65)$ & $1,453.300(455.26)$ & $<0.001$ \\
LLL_minimum & $1,093.485(171.46)$ & $1,344.760(425.07)$ & $<0.001$ \\
Run percentage & $0.580(0.10)$ & $85.705(34.94)$ & $<0.001$ \\
Run length non-uniformity & $70.024(27.21)$ & 0.0011 \\
\hline
\end{tabular}

$\mathrm{P}<0.05$ was considered to indicate a statistically significant difference. LLL, low frequency-low frequency-low frequency output.

compared with the other groups. Compared to the adenocarcinoma group, the SCLC group showed significantly increased texture parameters; similar results were observed between the squamous cell carcinoma and SCLC groups.

\section{Discussion}

In light of recent advances in the acquisition and analysis of MR images for the high-throughput extraction of imaging feature information, medical imaging can now be used to quantify the distinguishing features of tumor tissues (30-32). This approach is distinct from prior subjective or qualitative methods (14). Applying TA to medical imaging features has been a significant field of research and has generated an extensive body of literature (33-35). It has been demonstrated that MR images include tissue-specific texture features that can be extracted by mathematical methods (30). TA can be used to distinguish pathological from healthy human cerebral tissue and to classify different types of cerebral tissue $(15,36,37)$. TA enables the discrimination of multiple sclerosis lesions from normal and normal-appearing white matter (38). However, previous studies have mainly focused on detecting small nodules, that is, the lesions themselves. This study is distinct in that it primarily characterizes early cerebral tissue damage caused by lung cancer before metastasis by applying TA methods. The TA of MRI features can also be used for classifying different cerebral tissues and structures in brain MR images $(36,39)$.

MR images were selected in the current study to detect textural differences in cerebral tissue between patients with primary lung cancer and healthy controls. Of a total of 32 texture parameters tested, 27 parameters were significantly different between patients with lung cancer and healthy controls. The results of this study indicate that in patients with lung cancer, there are differences in the texture parameters of brain MR images of cerebral tissues. It can therefore be inferred that lung cancer can cause early brain tissue damage prior to metastasis.

Among the 27 significantly different parameters, six texture features that best discriminated between patients and healthy controls were identified by calculating Fisher coefficients. These texture parameters were the LLL_median, mean, LLL_ percentile, LLL_minimum, run percentage and run length non-uniformity. Using these six parameters, the two cerebral hemispheres and gray and white matter were compared between and within the participant groups. First, the left and right hemispheres of cerebral tissue were compared in the controls, as well as the gray and white matter. It is well known that the human brain is symmetrical in structure and with respect to gray and white matter, there are obvious differences in both composition and function. Gray matter is mainly composed of the cell bodies of neurons, but white matter is principally surrounded by the myelin sheaths of neurons. In regard to functionality, the former contains nerve centers, which play a major role in generating neural activity; the latter, on the other hand, represents the main transmission pathways carrying the neural signals generated by the brain and the gray matter of the spinal cord $(40,41)$. The results of the present study show that the texture parameters were not significantly different between the 2 hemispheres, but 4 of 6 texture parameters were significantly different between gray and white matter. It can therefore be concluded that TA applied to brain MR images is stable and reliable. This analysis was repeated in the patient group and an identical trend was observed, which may indicate that primary lung lesions can cause similar early damage in both hemispheres and that TA can be used to classify different structures. By comparison of different regions of gray and white matter between patients with lung cancer and healthy controls, all six texture parameters were significantly different in both gray matter and white matter. It can be assumed that the overt texture changes are caused by the lung cancer and this leads to similar impairments in both gray and white matter.

Clinical stage is used to describe the severity and range of involvement of malignant tumors according to the original tumor and degree of spread in an individual, which is the basis for understanding the extent of the disease. This information can help doctors develop appropriate treatment plans and understand the prognosis and outcome of the disease. The numerically higher the stage, the more advanced the tumor progression and the worse the prognosis (42). The present study analyzed associations between the clinical stages of lung cancer and texture parameters of MR images. The results revealed that, compared with the early-stage group (stage I+II), the advanced-stage group (stage III+IV) showed a more significant increase in all six parameters. This may indicate that increased parameters are related to more advanced clinical stages of lung cancer.

In addition to the clinical stage, the choice of tumor treatment is also related to the pathological type of the 
tumor $(18,28,43-46)$. Associations with the histological type were also analyzed in this study. The results show that compared to adenocarcinoma group, the SCLC group showed significantly increased texture parameters; similar results were found between the squamous cell carcinoma and SCLC groups. However, the texture parameters assessed in the adenocarcinoma and squamous cell carcinoma groups were not significantly different. It is necessary to note that compared with adenocarcinoma and squamous cell carcinoma, SCLC has a higher degree of malignancy and a worse prognosis $(47,48)$. The significant differences in texture parameters between SCLC and adenocarcinoma or squamous cell carcinoma are possibly attributable to these differences. It can be assumed that greater texture changes are caused by greater degrees of malignancy.

There are several limitations of this study. Firstly, as this is a preliminary experiment, the sample size was relatively small. Furthermore, a limited set of features, rather than a larger set of multiple hundreds or thousands (compound) of features was selected for analysis because the selected texture features have consistently been shown to be effective in the analysis of medical images $(25,38,49-51)$ and robust against variations between scanners and protocol parameters (52) especially after normalization (53). Finally, in the absence of confirmation of the early damage to human brain tissue, further pathological investigation using an animal model is required.

In conclusion, this small pilot study indicates that TA with a variable set of texture features can serve as an adjuvant diagnostic tool to traditional MRI to detect early damage in patients with lung cancer without brain metastasis. TA may serve as a new method for the study of tumor metastasis and paraneoplastic syndromes.

\section{Acknowledgements}

Not applicable.

\section{Funding}

The present study was funded by the National Natural Science Foundation of China (grant no. 81670046).

\section{Availability of data and materials}

The datasets used and/or analyzed during the current study are available from the corresponding author on reasonable request.

\section{Authors' contributions}

JX acquired the data, performed the analyses and wrote the initial manuscript. XC and BW performed texture analysis. GW, MH, RL and YQ acquired the data. JX and QY selected ROIs of the MR images. ZL and MH made substantial contributions to the conception and design of the present study. All authors read and approved the final manuscript.

\section{Ethical approval and consent to participate}

The present study was approved by the Ethics Committee of Shandong Provincial Hospital Affiliated to Shandong 
University (Jinan, China). Written informed consent was obtained from all patients prior to the study start.

\section{Patient consent for publication}

All patients signed consent for publication.

\section{Competing interests}

The authors declare that they have no competing interests.

\section{References}

1. Bray F, Ferlay J, Soerjomataram I, Siegel RL, Torre LA and Jemal A: Global cancer statistics 2018: GLOBOCAN estimates of incidence and mortality worldwide for 36 cancers in 185 countries. CA Cancer J Clin 68: 394-424, 2018.

2. Chen W, Sun K, Zheng R, Zeng H, Zhang S, Xia C, Yang Z, Li H, Zou X and He J: Cancer incidence and mortality in China, 2014. Chin J Cancer Res 30: 1-12, 2018.

3. Masters GA, Temin S, Azzoli CG, Giaccone G, Baker S Jr., Brahmer JR, Ellis PM, Gajra A, Rackear N, Schiller JH, et al: Systemic therapy for stage iv non-small-cell lung cancer: American Society of clinical oncology clinical practice guideline update. J Clin Oncol 33: 3488-3515, 2015.

4. Sul J and Posner JB: Brain metastases: Epidemiology and pathophysiology. Cancer Treat Res 136: 1-21, 2007.

5. Langley RR and Fidler IJ: The seed and soil hypothesis revisited-the role of tumor-stroma interactions in metastasis to different organs. Int J Cancer 128: 2527-2535, 2011.

6. Landis SH, Murray T, Bolden S and Wingo PA: Cancer statistics, 1998. CA Cancer J Clin 48: 6-29, 1998

7. Sawaya R: Considerations in the diagnosis and management of brain metastases. Oncology 15: 1145-1163, 2001.

8. Leypoldt F and Wandinger KP: Paraneoplastic neurological syndromes. Clin Exp Immunol 175: 336-348, 2014.

9. Gassmann P, Haier J, Schlüter K, Domikowsky B, Wendel C, Wiesner U, Kubitza R, Engers R, Schneider SW, Homey B and Müller A: CXCR4 regulates the early extravasation of metastatic tumor cells in vivo. Neoplasia 11: 651-661, 2009.

10. Erler JT, Bennewith KL, Cox TR, Lang G, Bird D, Koong A, Le QT and Giaccia AJ: Hypoxia-induced lysyl oxidase is a critical mediator of bone marrow cell recruitment to form the premetastatic niche. Cancer Cell 15: 35-44, 2009.

11. Erler JT and Weaver VM: Three-dimensional context regulation of metastasis. Clin Exp Metastasis 26: 35-49, 2009.

12. Hiratsuka S, Goel S, Kamoun WS, Maru Y, Fukumura D, Duda DG and Jain RK: Endothelial focal adhesion kinase mediates cancer cell homing to discrete regions of the lungs via E-selectin up-regulation. Proc Natl Acad Sci USA 108: 3725-3730, 2011.

13. Gupta GP and Massague J: Cancer metastasis: Building a framework. Cell 127: 679-695, 2006.

14. Aerts HJ, Velazquez ER, Leijenaar RT, Parmar C, Grossmann P, Carvalho S, Bussink J, Monshouwer R, Haibe-Kains B, Rietveld D, et al: Decoding tumour phenotype by noninvasive imaging using a quantitative radiomics approach. Nat Commun 5 : 4006, 2014

15. Abdullah N, Ngah UK and Aziz SA: Image classification of brain MRI using support vector machine. 2011 IEEE International Conference on Imaging Systems and Techniques, 242-247, 2011.

16. Connell JJ, Chatain G, Cornelissen B, Vallis KA, Hamilton A, Seymour L, Anthony DC and Sibson NR: Selective permeabilization of the blood-brain barrier at sites of metastasis. J Natl Cancer Inst 105: 1634-1643, 2013.

17. Holli KK, Harrison L, Dastidar P, Waljas M, Liimatainen S, Luukkaala T, Ohman J, Soimakallio S and Eskola H: Texture analysis of MR images of patients with mild traumatic brain injury. BMC Med Imaging 10: 8, 2010.

18. Travis WD, Brambilla E, Nicholson AG, Yatabe Y, Austin JHM, Beasley MB, Chirieac LR, Dacic S, Duhig E, Flieder DB, et al: The 2015 World Health organization classification of lung tumors: Impact of Genetic, Clinical and Radiologic Advances Since the 2004 Classification. J Thorac Oncol 10: 1243-1260, 2015.
19. Chernov MF, Nakaya K, Izawa M, Hayashi M, Usuba Y, Kato K, Muragaki Y, Iseki H, Hori T and Takakura K: Outcome after radiosurgery for brain metastases in patients with low Karnofsky performance scale (KPS) scores. Int_J Radiat Oncol Biol Phys 67: 1492-1498, 2007.

20. Karcioglu O, Topacoglu H, Dikme O and Dikme O: A systematic review of the pain scales in adults: Which to use? Am J Emerg Med 36: 707-714, 2018.

21. Zimny A, Szmyrka-Kaczmarek M, Szewczyk P, Bladowska J, Pokryszko-Dragan A, Gruszka E, Wiland P and Sasiadek M: In vivo evaluation of brain damage in the course of systemic lupus erythematosus using magnetic resonance spectroscopy, perfusion-weighted and diffusion-tensor imaging. Lupus 23: $10-19,2014$

22. Bladowska J, Zimny A, Knysz B, Malyszczak K, Koltowska A, Szewczyk P, Gasiorowski J, Furdal M and Sasiadek MJ: Evaluation of early cerebral metabolic, perfusion and microstructural changes in HCV-positive patients: A pilot study. J Hepatol 59: 651-657, 2013.

23. Loizou CP, Petroudi S, Seimenis I, Pantziaris M and Pattichis CS: Quantitative texture analysis of brain white matter lesions derived from T2-weighted MR images in MS patients with clinically isolated syndrome. J Neuroradiol 42: 99-114, 2015.

24. Mayerhoefer ME, Szomolanyi P, Jirak D, Berg A, Materka A, Dirisamer A and Trattnig S: Effects of magnetic resonance image interpolation on the results of texture-based pattern classification: a phantom study. Invest Radiol 44: 405-411, 2009.

25. Becker AS, Schneider MA, Wurnig MC, Wagner M, Clavien PA and Boss A: Radiomics of liver MRI predict metastases in mice. Eur Radiol Exp 2: 11, 2018.

26. Yao J, Dwyer A, Summers RM and Mollura DJ: Computer-aided diagnosis of pulmonary infections using texture analysis and support vector machine classification. Acad Radiol 18: 306-314, 2011.

27. van Griethuysen JJM, Fedorov A, Parmar C, Hosny A, Aucoin N, Narayan V, Beets-Tan RGH, Fillion-Robin JC, Pieper S and Aerts HJWL: Computational radiomics system to decode the radiographic phenotype. Cancer Res 77: e104-e107, 2017.

28. Nasim F, Sabath BF and Eapen GA: Lung Cancer. Med Clin North Am 103: 463-473, 2019.

29. Kay FU, Kandathil A, Batra K, Saboo SS, Abbara S and Rajiah P: Revisions to the Tumor, Node, Metastasis staging of lung cancer (8th edition): Rationale, radiologic findings and clinical implications. World J Radiol 9: 269-279, 2017.

30. Castellano G, Bonilha L, Li LM and Cendes F: Texture analysis of medical images. Clin Radiol 59: 1061-1069, 2004.

31. Napel S, Mu W, Jardim-Perassi BV, Aerts HJWL and Gillies RJ: Quantitative imaging of cancer in the postgenomic era: Radio(geno)mics, deep learning, and habitats. Cancer 124: 4633-4649, 2018.

32. Gallego-Ortiz C and Martel AL: Using quantitative features extracted from T2-weighted MRI to improve breast MRI computer-aided diagnosis (CAD). PLoS One 12: e0187501, 2017.

33. Nachimuthu DS and Baladhandapani A: Multidimensional texture characterization: On analysis for brain tumor tissues using MRS and MRI. J Digit Imaging 27: 496-506, 2014.

34. Galm BP, Martinez-Salazar EL, Swearingen B, Torriani M, Klibanski A, Bredella MA and Tritos NA: MRI texture analysis as a predictor of tumor recurrence or progression in patients with clinically non-functioning pituitary adenomas. Eur J Endocrinol 179: 191-198, 2018.

35. Holli-Helenius K, Salminen A, Rinta-Kiikka I, Koskivuo I, Bruck N, Bostrom P and Parkkola R: MRI texture analysis in differentiating luminal A and luminal B breast cancer molecular subtypes-a feasibility study. BMC Med Imaging 17: 69, 2017.

36. Herlidou-Meme S, Constans JM, Carsin B, Olivie D, Eliat PA, Nadal-Desbarats L, Gondry C, Le Rumeur E, Idy-Peretti I and de Certaines JD: MRI texture analysis on texture test objects, normal brain and intracranial tumors. Magn Reson Imaging 21: 989-993, 2003.

37. Kjaer L, Ring P, Thomsen $\mathrm{C}$ and Henriksen O: Texture analysis in quantitative MR imaging. Tissue characterisation of normal brain and intracranial tumours at $1.5 \mathrm{~T}$. Acta Radiol 36: 127-135, 1995.

38. Mougiakakou SG, Valavanis IK, Nikita A and Nikita KS: Differential diagnosis of CT focal liver lesions using texture features, feature selection and ensemble driven classifiers. Artif Intell Med 41: 25-37, 2007. 
39. Lerski RA, Straughan K, Schad LR, Boyce D, Bluml S and Zuna I: MR image texture analysis-an approach to tissue characterization. Magn Reson Imaging 11: 873-887, 1993.

40. Cristofori I, Zhong W, Chau A, Solomon J, Krueger F and Grafman J: White and gray matter contributions to executive function recovery after traumatic brain injury. Neurology 84 : 1394-1401, 2015

41. Barbey AK, Colom R, Solomon J, Krueger F, Forbes C and Grafman J: An integrative architecture for general intelligence and executive function revealed by lesion mapping. Brain 135: 1154-1164, 2012.

42. Woodard GA, Jones KD and Jablons DM: Lung cancer staging and prognosis. Cancer Treat Res 170: 47-75, 2016.

43. Derks JL, Leblay N, Thunnissen E, van Suylen RJ, den Bakker M, Groen HJM, Smit EF, Damhuis R, van den Broek EC, Charbrier A, et al: Molecular subtypes of pulmonary large-cell neuroendocrine carcinoma predict chemotherapy treatment outcome. Clin Cancer Res 24: 33-42, 2018.

44. Schwartz AM and Rezaei MK: Diagnostic surgical pathology in lung cancer: Diagnosis and management of lung cancer, 3rd ed: American College of Chest Physicians evidence-based clinical practice guidelines. Chest 143 (5 Suppl): e251S-e262S, 2013.

45. Travis WD, Brambilla E, Noguchi M, Nicholson AG, Geisinger KR, Yatabe Y, Beer DG, Powell CA, Riely GJ, Van Schil PE, et al: International association for the study of lung cancer/american thoracic society/european respiratory society: International multidisciplinary classification of lung adenocarcinoma: Executive summary. Proc Am Thorac Soc6: 244-285, 2011.

46. Travis WD, Brambilla E and Riely GJ: New pathologic classification of lung cancer: Relevance for clinical practice and clinical trials. J Clin Oncol 31: 992-1001, 2013.

47. Blandin Knight S, Crosbie PA, Balata H, Chudziak J, Hussell T and Dive C: Progress and prospects of early detection in lung cancer. Open Biol 7: pii: 170070, 2017.
48. Walters S, Maringe C, Coleman MP, Peake MD, Butler J, Young N, Bergstrom S, Hanna L, Jakobsen E, Kolbeck K, et al: Lung cancer survival and stage at diagnosis in Australia, Canada, Denmark, Norway, Sweden and the UK: A population-based study, 2004-2007. Thorax 68: 551-564, 2013.

49. Dennie C, Thornhill R, Sethi-Virmani V, Souza CA, Bayanati H, Gupta A and Maziak D: Role of quantitative computed tomography texture analysis in the differentiation of primary lung cancer and granulomatous nodules. Quant Imaging Med Surg 6: 6-15, 2016.

50. Wibmer A, Hricak H, Gondo T, Matsumoto K, Veeraraghavan $\mathrm{H}$, Fehr D, Zheng J, Goldman D, Moskowitz C, Fine SW, et al: Haralick texture analysis of prostate MRI: Utility for differentiating non-cancerous prostate from prostate cancer and differentiating prostate cancers with different Gleason scores. Eur Radiol 25: 2840-2850, 2015.

51. MacKay JW, Murray PJ, Low SB, Kasmai B, Johnson G, Donell ST and Toms AP: Quantitative analysis of tibial subchondral bone: Texture analysis outperforms conventional trabecular microarchitecture analysis. J Magn Reson Imaging 43: 1159-1170, 2016.

52. Mayerhoefer ME, Szomolanyi P, Jirak D, Materka A and Trattnig S: Effects of MRI acquisition parameter variations and protocol heterogeneity on the results of texture analysis and pattern discrimination: An application-oriented study. Med Phys 36: 1236-1243, 2009.

53. Collewet G, Strzelecki M and Mariette F: Influence of MRI acquisition protocols and image intensity normalization methods on texture classification. Magn Reson Imaging 22: 81-91, 2004.

This work is licensed under a Creative Commons Attribution-NonCommercial-NoDerivatives 4.0 International (CC BY-NC-ND 4.0) License. 